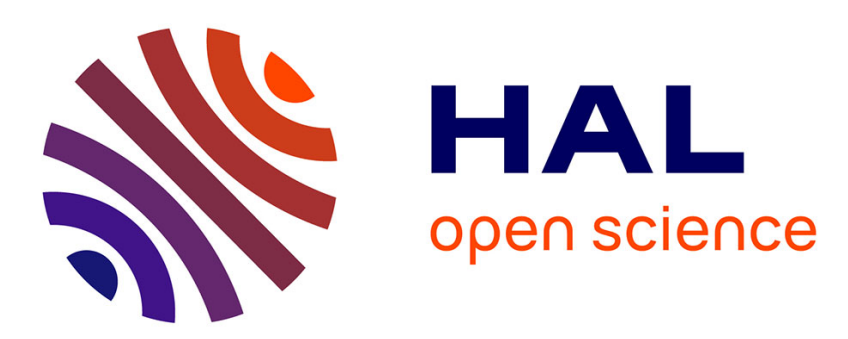

\title{
Focusing double bent crystal diffractometer in combination with PSD for SANS experiments
}

\author{
J. Saroun, P. Lukas, P. Mikula, P. Strunz
}

\section{To cite this version:}

J. Saroun, P. Lukas, P. Mikula, P. Strunz. Focusing double bent crystal diffractometer in combination with PSD for SANS experiments. Journal de Physique IV Proceedings, 1993, 03 (C8), pp.C8-439-C8442. 10.1051/jp4:1993890 . jpa-00252321

\section{HAL Id: jpa-00252321 https://hal.science/jpa-00252321}

Submitted on 1 Jan 1993

HAL is a multi-disciplinary open access archive for the deposit and dissemination of scientific research documents, whether they are published or not. The documents may come from teaching and research institutions in France or abroad, or from public or private research centers.
L'archive ouverte pluridisciplinaire HAL, est destinée au dépôt et à la diffusion de documents scientifiques de niveau recherche, publiés ou non, émanant des établissements d'enseignement et de recherche français ou étrangers, des laboratoires publics ou privés. 


\title{
Focusing double bent crystal diffractometer in combination with PSD for SANS experiments
}

\author{
J. ŠAROUN, P. LUKÁŠ, P. MIKULA and P. STRUNZ
}

Nuclear Physics Institute, 25068 Řě near Prague, Czech Republic

\begin{abstract}
A new concept of medium resolution SANS measurements in a double bent crystal arrangement using fully asymmetric analyzer geometry was suggested earlier. This setup enables a positional analysis of the scattering curve and thus, to collect the whole spectrum simultaneously by a linear position sensitive detector (PSD). It is shown, on the basis of both calculations and Monte Carlo simulations, that the neutron beam can be focused at the PSD, which practically leads to the gain in angular resolution. These theoretical predictions are proved experimentally.
\end{abstract}

\section{Introduction}

A double perfect crystal setting in Bragg reflection geometry provides very narrow rocking curves ( $\left.\delta Q \sim 2 \cdot 10^{-5} \AA^{-1}\right)$, which makes this arrangement convenient for small-angle neutron scattering (SANS) measurements with a very high resolution, however at the cost of ar: exceedingly low neutron intensity [1]. The luminosity of the double crystal diffractometer can be increased if elastically bent perfect crystals are used. SANS measurements in a medium resolution range $\delta Q=\left(2 \cdot 10^{-4}-2 \cdot 10^{-3}\right) \AA^{-1}$ can then be performed more easily [2]. Furthemore, since the orientation of diffraction planes depends monotonously on the position along the bent crystal slab, the use of fully asymmetric diffraction geometry of the analyzer enables us to collect the whole spectrum simultaneously by a position sensitive detector [3,4]. For a further development of this experimental technique it was necessary to study the optical properties of the double bent crystal (DBC) setting in more detail. The contributions of the crystal thicknesses and the beam collimation to the fina: angular resolution were calculated on the basis of statistica? considerations [5]. The obtained results were checked by both Monte Carlo simulations and experiments.

\section{Focusing Double Crystal Arrangement}

The scheme of the focusing DBC difractometer used for SANs experiments is shown in Fig. 1 . 


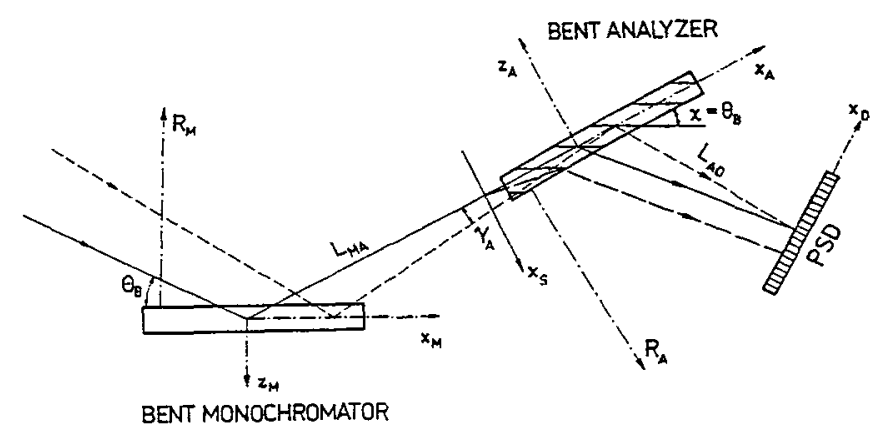

Fig. 1 Schematic sketch of the focusing DBC diffractometer and neutron trajectories. An arbitrary unscattered neutron $(-\ldots)$ crosses the central beam (- - ) at the detector, while the scattered neutrons (- - ) reach the detector at the distance $x_{p}$ according to eq. (2).

Let us consider positive monochromator and negative analyzer curvatures, a narrow slit at the sample position and an arbitrary neutron trajectory (dashed line). Due to the bending, the neutron is reflected with a slightly lower Bragg angle. Consequently, it meets the Bragg condition further inside the analyzer and then crosses the cen-tral beam at a certain distance. Thus, the divergent beam can be fo.cused at the detector, which practically improves the angular resolution of the diffractometer, since the peak position at the detector is obviously related to the analyzer inclination $\vartheta$ by the equation

$$
\mathbf{x}_{D}=\vartheta \mathrm{R}_{\mathrm{A}} \sin \left(2 \Theta_{B}\right),
$$

where $R_{A}$ is the analyzer radius of curvature. Similarly, the wide parallel beam can also be focused at the same distance if a proper ratio between crystal curvatures is chosen. These focusing conditions correspond to a situation when the beam collimation does not influence the angular resolution. The collimation contributions to the resolution were calculated on the basis of statistical consider ations by the method of Popovici et al. [5]. The optimal analyzer curvature and detector distance could then be determined as [6]

$$
\begin{aligned}
\mathrm{L}_{A D} & =\mathrm{K}\left(\Theta_{B}\right) \mathrm{L}_{M A} \\
\mathrm{R}_{A} \sin \left(2 \Theta_{B}\right) & =-\mathrm{K}\left(\Theta_{B}\right) \mathrm{R}_{\mathrm{H}} \sin \left(\Theta_{B}\right),
\end{aligned}
$$

which holds for small crystal curvatures $\left(R_{M, A} \gg L_{M A}\right)$ and the same diffraction planes of both crystals. Here $L_{M A}$ is the distance betwaen the crystals and $K(\oplus)$ is the function depending only on the Bragg angle and the Poisson constant $v$, as

$$
\mathrm{K}(\Theta)=1+4\left((1+v) \cos ^{2} \Theta-1\right) \sin ^{2} \Theta .
$$

The instrumental curves of the focusing DBC diffractometer were simulated in dependence on the analyzer curvature for two different beam divergences. The angular resolution was then characterized by the angular dispersions $\left\langle\vartheta^{2}\right\rangle^{1 / 2}$ of the instrumental curves (Fig. 2). 

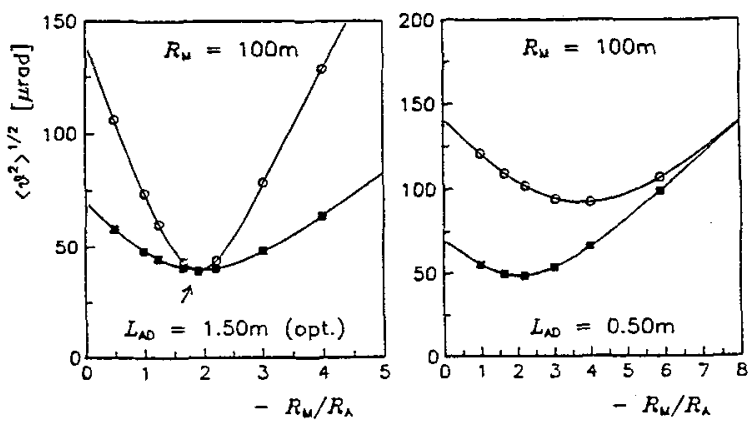

Fig. 2 The dependence of the angular resolution on the analyzer curvature, simulated (points) and calculated (lines) for different beam divergences $\left(\Delta \gamma=6.4^{\prime}(-)\right.$ and $\left.26^{\prime}(0)\right)$ and detector distances. The setting independent on the collimation is denoted by the arrow.

It may be seen that the angular resolution does not depend on the beam collimation for the optimal choice of the crystal bending ratio and the detector distance.

\section{Experimental}

The experimental testing was performed at the SANs diffractometer at Nuclear Physics Institute (NPI) in Řež [4], with Si(111) reflections for both crystals $\left(\Theta_{B}=19.5^{\circ}\right)$, sample slit width $7 \mathrm{~mm}$ and $\mathrm{L}_{\mathrm{MA}}=1.65 \mathrm{~m}$. The beam profiles were measured at differen distances from the analyzer (Fig. 3). The focusing effect was observed even though the beam divergence was rather low $\left(\Delta \gamma=15^{\prime}\right)$. The effect would have been more apparent with a more divergent beam since the angular resolution of the optimal arrangement depends (in spite of: the detector resolution) only on the thickness and curvature of the monochromator. Outside of this optimum, the beam profile is nore influenced by the collimation, which results in the observed peak asymmetry.
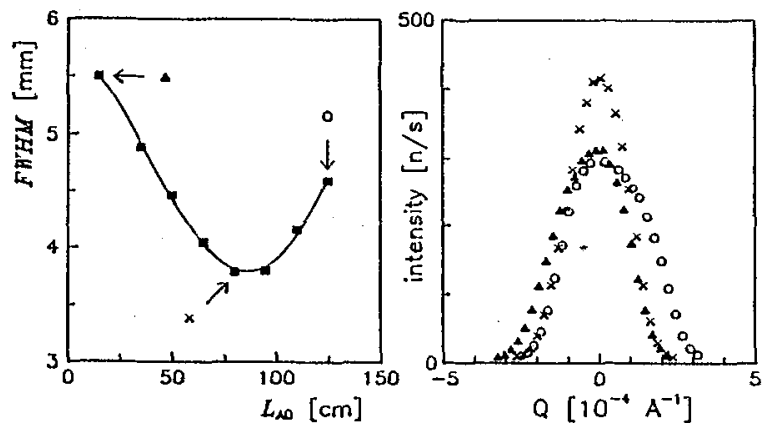

Fig. 3 The dependence of beam profiles on the detector distance (experimental data). The zero on the q-scale was determined as the apex of the curves.

The next experiment was performed with a standard sample, the 1. $3 \mathrm{~mm}$ thick polyethylene plate filled with graphite, which gives rise to the sans with the total probability about $2 \%$. This sample was 
measured at both, the focusing diffractometer employing PSD and the conventional DBC diffractometer (SPN-100 at NPI Rež [2]). The data. were collected with similar resolutions and expositions (Fig.4). These results clearly illustrate the advantage of using the focusing asymmetric DBC arrangement.
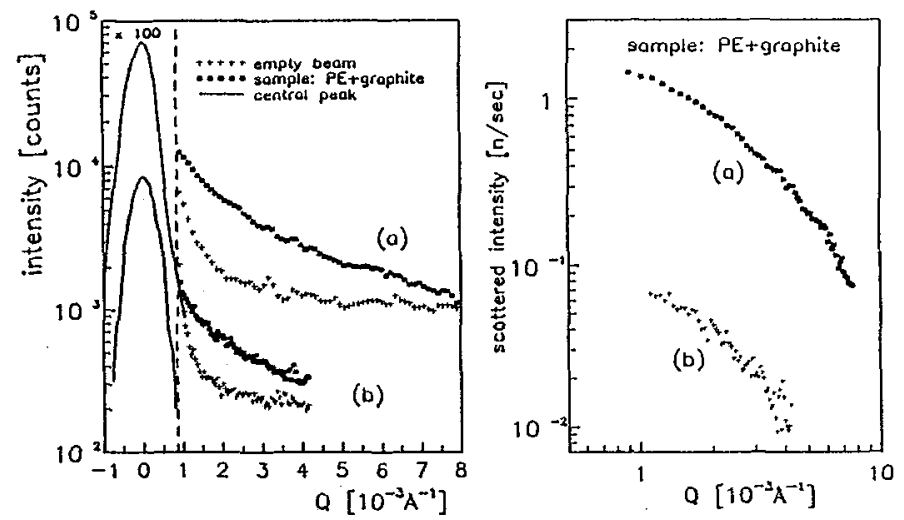

Fig. 4 Comparison of SANS measurements in the focusing arrangement using the PSD (a) and the conventional step-by-step measurement in symmetric reflection geometry (b) at the standard sample ( $\mathrm{PE}+$ graphite). In the right figure, the subtracted scattered intensity related to one second of the total exposition time is plotted.

(a) resolution $6.9 \cdot 10^{-4} \AA^{-1}$, exposition $2.8 \mathrm{~h}$

\section{Conclusions}

The advantage of using the DBC arrangement in fully asymmetric geometry for SANS experiments obviously consists in the possibility of simultaneous data acquisition. Moreover, it was found that the optical properties of bent single crystals enable spatial bean focusing to improve the angular resolution. Under the optimal experimental conditions defined by eqs.(2), the instrumental peak profile does not depend on the beam collimation. Therefore a highly divergent neutron beam can be exploited in the experiment. In comparison to the step-by-step measurements in conventional symmetric reflection geometry, the exposition time can be substantialy reduced, which makes the new focusing diffractometer suited even for in situ measurements.

\section{References}

[1] SCHWAHN D., MIKŠOVSKY A., RAUCH H., SEIDL E. and ZUGAREK G., Nucl. Instr. and Methods A239 (1985) $229-234$.

[2] KULDA J. and MIKULA P., J. AppI. Cryst. 16 (1983) 498-504.

[3] MIKULA P., LUKAS P. and EICHHORN F., J.Appl. Cryst. 21 (1988) 981-983.

[4] LUKAS P., MIKULA P., KULDA J., SAROUN J. and STRUNZ P., Physicá B $180 \& 181$ (1992) 984-986.

[5] POPOVICI M., STOICA A. D., CHALUPA B. and MIKULA P. J. App1. Cryst. 21 (1988) 958-965.

[6] SAROUN J., LUKÁS P., MIKULA P. and ALEEELD B. (1993), submit:ed to J. Appl. Cryst. 